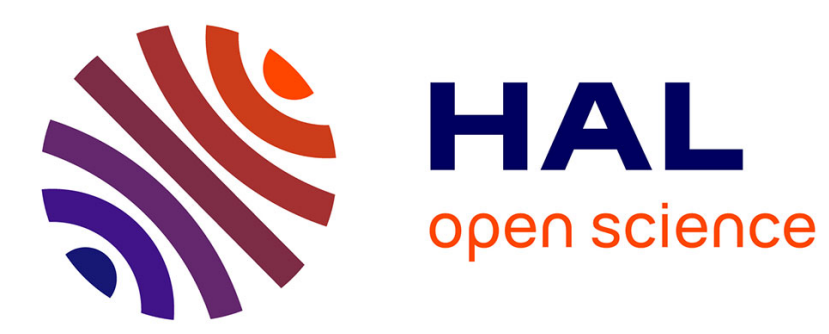

\title{
Les conventions internationales sur l'environnement: état des ratifications et des engagements des pays développés et des pays en développement
}

\author{
Moise Tsayem Demaze
}

\section{- To cite this version:}

Moise Tsayem Demaze. Les conventions internationales sur l'environnement: état des ratifications et des engagements des pays développés et des pays en développement. L'Information géographique, 2009, 73 (3), pp.84-99. halshs-00420875

\section{HAL Id: halshs-00420875 \\ https://shs.hal.science/halshs-00420875}

Submitted on 29 Sep 2009

HAL is a multi-disciplinary open access archive for the deposit and dissemination of scientific research documents, whether they are published or not. The documents may come from teaching and research institutions in France or abroad, or from public or private research centers.
L'archive ouverte pluridisciplinaire HAL, est destinée au dépôt et à la diffusion de documents scientifiques de niveau recherche, publiés ou non, émanant des établissements d'enseignement et de recherche français ou étrangers, des laboratoires publics ou privés. 


\title{
Doc IG
}

\section{Les conventions internationales sur l'environnement : état des ratifications et des engagements des pays développés et des pays en développement}

\author{
Moïse Tsayem Demaze \\ Université du Maine, UMR CNRS 6590 ESO-GREGUM \\ Chercheur associé à l'IRD, US 140 ESPACE \\ Avenue Olivier Messiaen, 72085 Le Mans Cedex 9 \\ Tél. 02.43.83.31.46, Fax 0243833192 \\ Moise.Tsayem_Demaze@univ-lemans.fr
}

\begin{abstract}
Résumé
Dans le sillage du sommet mondial sur l'environnement et le développement organisé en 1992 à Rio de Janeiro au Brésil, des conventions internationales ont été adoptées pour fixer le cadre et pour définir les actions à mener en vue de résoudre les problèmes environnementaux globaux : réchauffement du climat, érosion de la biodiversité, sécheresse et désertification. Ce document présente ces conventions en les situant dans le contexte des principaux enjeux environnementaux mondiaux et en faisant un bilan des engagements pris par les pays développés et par les pays en développement. Les cartes réalisées à partir de l'état des ratifications au début de l'année 2008 montrent que ces conventions ont été ratifiées par la quasi-totalité des pays. La non ratification du protocole de Kyoto et de la convention sur la diversité biologique par les Etats-Unis est notable, tout comme la faible adhésion des pays au protocole de Carthagène. Bien que ratifiée par presque tous les pays, la convention sur la sécheresse et la désertification semble susciter moins d'intérêt et d'effervescence politique par rapport aux autres conventions et protocoles. Une synthèse cartographique propose une analyse comparative des ratifications de l'ensemble des traités, ce qui permet de se rendre compte de la mobilisation différenciée des Etats en vue de résoudre les problèmes environnementaux internationaux.
\end{abstract}

Mots-clés : conventions internationales, protocoles, changement climatique, biodiversité, sécheresse, désertification, environnement.

\section{Abstract. International conventions on environement : ratifications and commitments of developed and undeveloped countries}

In the framework of the world summit on environment and development organized in 1992 in Rio de Janeiro, Brazil, international conventions were adopted to define actions to be carried out in order to solve global environmental issues : climate change, biodiversity loss, dryness and desertification. This paper presents these conventions and specifies the context and the stake of the world main environmental problems. Commitments of developed countries and undeveloped countries are analysed. The maps we drawn according to the state of ratifications at the beginning of the year 2008 show that near total of the countries adhered to these conventions. The lack of ratification of the Kyoto protocol and the convention on biological diversity by United States is notable, like the weak adhesion of countries to the Cartagena protocol as well as the little interest and the weak political mobilization for the convention on dryness and desertification. A cartographic synthesis proposes a comparative analysis of the ratifications of these conventions and protocols. This final map makes it possible to evaluate differences in States mobilization in order to solve international environmental problems.

Keys-words: international conventions, protocols, climate change, biodiversity, dryness, desertification, environment. 


\section{Introduction}

La conférence organisée par les Nations unies à Rio de Janeiro en 1992 a érigé les questions d'environnement et de développement aux premiers rangs des préoccupations de la communauté internationale. Baptisée sommet de la Terre, cette conférence a réaffirmé le caractère planétaire des problématiques de dégradation des écosystèmes et de gestion des ressources naturelles dans la perspective du développement durable.

En soulignant la dimension planétaire ou globale des problèmes d'environnement, la conférence de Rio a largement contribué à l'émergence du droit international de l'environnement qui comporte plusieurs conventions (accords officiels entre États) dont l'objectif est de régir le traitement des questions environnementales globales. Les conventions environnementales qui mobilisent le plus la communauté internationale actuellement sont celles qui sont issues directement du sommet de Rio. Il s'agit de la convention sur les changements climatiques et de la convention sur la diversité biologique.

A ces deux conventions majeures est généralement associée la convention sur la lutte contre la désertification dans les pays gravement touchés par la sécheresse. Cette convention sur la désertification a été élaborée en 1994 à Paris et s'inscrit dans le cadre des actions prônées par la Conférence de Rio.

Il existe quelques autres conventions qui sont antérieures à Rio et qui témoignent des préoccupations écologiques internationales notamment à partir des années 1970. Ces conventions pré-Rio concernent les zones humides (Ramsar, 1971), le commerce international des espèces de faune et de flore sauvages menacées d'extinction (CITES, 1973), la protection du patrimoine mondial culturel et naturel (Paris, UNESCO, 1972), la conservation de la vie sauvage et du milieu naturel de l'Europe (Berne, 1979), etc.

Ce document ne traite que des conventions issues directement ou indirectement du sommet de Rio. Il présente d'abord la convention sur les changements climatiques et le protocole de Kyoto, puis la convention sur la diversité biologique et le protocole de Carthagène. Ces quatre traités internationaux sont directement issus du sommet de Rio. La convention sur la désertification, discutée à Rio mais finalisée à Paris en 1994, est présentée ensuite. Le contenu de chaque convention ou protocole est résumé, et les éléments essentiels sont évoqués. Le bilan des ratifications de chaque traité est illustré cartographiquement. Chaque carte a été réalisée à partir d'informations mises à jour en janvier $2008^{1}$. La dernière partie de ce document propose une synthèse cartographique qui sert de support pour une analyse comparative des ratifications de l'ensemble des conventions et protocoles. Cette carte finale permet de se rendre compte de la mobilisation différenciée des Etats. Du fait des engagements qui leur sont peu contraignants, les pays en développement ont ratifié généralement la quasitotalité des traités, alors que des pays développés, comme le Canada ou les Etats-Unis, n’ont pas ratifié des traités qui leur paraissent très contraignants. La géographie des ratifications ainsi esquissée suggère la difficulté d'amener l'ensemble des Etats à participer aux efforts à fournir pour résoudre des problèmes environnementaux pourtant très souvent présentés comme étant internationaux et graves pour l'humanité.

\footnotetext{
${ }^{1}$ Ces informations sont diffusées par le Programme des Nations unies pour l'Environnement (PNUE) qui coordonne les secrétariats de ces principales conventions environnementales internationales. Ces conventions et protocoles sont consultables sur le site http://www.unep.org/science/fr/secretariat/index.asp
} 


\section{La convention sur le changement climatique : la mise en cause des pays développés}

Cette convention concrétise la prise de conscience de la communauté internationale sur les risques et les capacités de modification du climat du fait des activités humaines. Dès l'article 1 de la convention, les changements climatiques sont définis comme étant « des changements de climat qui sont attribués directement ou indirectement à une activité humaine altérant la composition de l'atmosphère mondiale et qui viennent s'ajouter à la variabilité naturelle du climat» (art.1, alinéa 2). Cette définition souligne l'enjeu de la préoccupation de la communauté internationale, car l'inquiétude vient du fait qu'au-delà de la variabilité naturelle du climat, les activités humaines sont considérées comme étant désormais capables de perturber le fonctionnement du système climatique.

A la suite de cette perception d'un possible dérapage climatique du fait de l'Homme, l'article 2 de la convention définit l'objectif à atteindre : "stabiliser, conformément aux dispositions pertinentes de la convention, les concentrations de gaz à effet de serre dans l'atmosphère à un niveau qui empêche toute perturbation anthropique dangereuse du système climatique ». Cet article ajoute qu'il « conviendra d'atteindre ce niveau dans un délai qui soit suffisant pour que les écosystèmes puissent s'adapter naturellement aux changements climatiques, que la production alimentaire ne soit pas menacée et que le développement économique puisse se poursuivre d'une manière durable ». Il s'agit là d'une formulation diplomatique qui dissimule mal les contradictions et le flou théorique et conceptuel entre développement, préservation de l'environnement et développement durable.

L'alinéa 2 de l'article 4 précise que les pays développés ainsi que l'ensemble des pays figurant dans l'annexe I (pays d'Europe centrale et orientale) doivent prendre des engagements spécifiques contenant des mesures et politiques visant à réduire leurs émissions de gaz à effet de serre pour les ramener à leur niveau de 1990. Ces pays développés doivent en outre fournir des « ressources financières nouvelles et additionnelles pour couvrir la totalité des coûts convenus encourus par les pays en développement » du fait de l'exécution de leurs obligations conformément à la convention (art. 4, alinéa 3). En d'autres termes, l'application de la convention par les pays en développement est dépendante des crédits alloués à cet effet par les pays riches. Ces derniers sont mis en cause du fait que leur développement, depuis la révolution industrielle, est accusé d'avoir entraîné un accroissement considérable des émissions et des concentrations de gaz à effet de serre dans l'atmosphère. Du fait de cette « responsabilité historique », il est demandé aux pays développés d'être à l'avant-garde de la lutte contre les changements climatiques. La convention évoque néanmoins les responsabilités « communes » de l'ensemble des pays, en ajoutant qu'elles sont «différenciées » suivant qu'il s'agit des pays développés (responsabilité élevée) ou des pays en développement (responsabilité faible). C'est sur cette base que l'effort demandé aux pays en développement est modulé (faible implication). Il est étonnant de constater que ces notions de « responsabilité historique » et de «responsabilités communes mais différenciées »n'ont pas suscité des débats, voire des réprobations, sur les plans juridiques, politiques et géopolitiques (Tsayem, 2009a et b).

Adoptée en 1992 à Rio et ouverte immédiatement aux signatures et ratifications, cette convention est entrée en application dans 190 pays (données de janvier 2008), soit la quasitotalité des pays du monde (fig. 1). Des pays en guerre, comme la Somalie et l'Iraq, figurent parmi les quelques pays qui ne l'ont pas ratifiée. Considérée comme un premier pas au niveau international, elle ne fixe pas d'engagements chiffrés à respecter par les pays. Cette insuffisance a été prise en compte dans le protocole de Kyoto. 


\section{Le Protocole de Kyoto : l'attribution des quotas en vue de réduire les émissions de gaz à effet de serre}

Ce protocole est le résultat d'âpres négociations politiques qui ont opposé principalement les États-Unis, l'Union européenne et les pays en développement. Adopté en 1997 et ouvert dès lors aux signatures et aux ratifications, son objectif est de limiter et de réduire les émissions de gaz à effet de serre en fixant des quotas d'émissions à respecter par les pays développés, considérés comme principaux responsables du changement climatique. Suivant l'esprit et les principes de la convention sur les changements climatiques, l'année 1990 est prise comme année de référence et le cap est fixé à 2012. A cette année butoir (2012), la production mondiale de gaz à effet de serre d'origine anthropique ne doit pas être supérieure à celle de 1990. La méthode choisie est l'attribution de quotas d'émissions globalement inférieurs de 5,2 \% par rapport au niveau d'émissions de 1990 (art. 3). La période 2008-2012 est retenue pour l'application de ces quotas répartis schématiquement ainsi qu'il suit :

- $8 \%$ de réduction pour les pays de l'Union européenne (comprenant alors 15 pays) avec des nuances tenant compte du niveau de pollution émise par pays ainsi que des progrès accomplis en matière de lutte contre la pollution. De la sorte, la France devra seulement stabiliser ses émissions (en raison de son choix pour l'utilisation de l'énergie nucléaire jugée non ou peu émettrice de gaz à effet de serre), tandis que l'Allemagne doit réduire ses émissions de $21 \%$ et que l'Espagne peut augmenter les siennes de $15 \%$ (du fait de son retard industriel);

- 7\% de réduction pour les États-Unis (qui ont signé le protocole mais ne l'ont pas ratifié, ce qui les affranchit de tout engagement contraignant) ;

- $6 \%$ de réduction pour le Japon ainsi que pour le Canada ;

- Statu quo pour la Russie et l'Ukraine (en raison de la récession industrielle récente dans ces deux pays) ;

- $8 \%$ d'augmentation pour l'Australie ;

- les pays en développement et les pays émergeants (Chine, Mexique, Brésil, etc.) sont exemptés de réduction contraignante.

La volonté de concilier les positions des uns et des autres et de trouver des compromis est transcrite dans le protocole au travers des «mécanismes de flexibilité » qui visent à faciliter l'acceptation politique du protocole et à réduire les coûts économiques qu'implique l'application des mesures adoptées. Ces mécanismes de flexibilité comprennent les échanges ou le commerce des droits d'émissions de gaz à effet de serre entre pays développés (marchés des quotas d'émissions ou permis transférables), la mise en œuvre conjointe entre pays de l'Europe du Centre et de l'Est, et enfin le mécanisme pour un développement «propre » (arrangements entre pays développés et pays en développement).

La mobilisation de la communauté internationale à travers le Protocole de Kyoto parait insuffisante dans la perspective d'une résorption de la «crise du climat » à l'horizon 2012. De nombreux experts trouvent que l'objectif de réduction globale de 5,2\% des émissions de gaz à effet de serre ne pourra pas permettre d'éviter le changement climatique. D'après les experts du GIEC, un objectif de réduction globale de 60 à $70 \%$ des émissions de gaz à effet de serre est plus à même de permettre de limiter efficacement le réchauffement de la Terre (3 3 ème rapport du GIEC). Durand (2002) indique que les 5,2\% de réduction préconisés par le 
protocole correspondraient à $0,06^{\circ} \mathrm{C}$, ce qui représente une baisse dérisoire (en moyenne 3 à $5 \%$ de l'effort total à fournir).

Les autres critiques que suscite le protocole de Kyoto concernent, entre autres :

- l'absence de sanctions applicables en cas de non respect des engagements pris par les États. Cette absence de sanctions soulève par ailleurs la question du suivi des décisions internationales face aux prérogatives des Etats ;

- la non implication de certains pays dans la lutte contre le réchauffement climatique, notamment les pays en développement (y compris les pays dits émergeants) jugés peu ou non responsables du réchauffement actuel.

- l'importance accordée aux priorités de développement économique et industriel par rapports aux impératifs écologiques. Ainsi, le protocole, comme la convention sur le climat, reconnaît que les pays en développement doivent s'occuper prioritairement de leur croissance économique et de la lutte contre la pauvreté. Or cela suppose un accroissement de leurs émissions de gaz carbonique. Tandis que les pays développés sont mis en cause du fait de leur « responsabilité historique », il est reconnu aux pays en développement la possibilité de se développer à leur tour même si cela doit aussi dégrader l'environnement et le climat. Le protocole est coincé entre logique économique (développement) et logique écologique (protection de l'environnement, développement durable). La logique économique semble même l'emporter sur la logique écologique comme en témoigne la création en 2005 des marchés carbone de l'Union Européenne et de la Banque Mondiale (Karsenty et Pirard, 2007a et b; Tsayem, 2008).

Les États-Unis, qui représentent environ $25 \%$ des émissions mondiales de gaz à effet de serre (et environ $5 \%$ de la population du monde), ont refusé de ratifier le protocole, arguant qu'il va à l'encontre de leurs intérêts économiques et industriels. La Russie a ratifié le protocole à la fin de l'année 2004, ce qui a permis son entrée en vigueur en février 2005. L'Union Européenne a ratifié le protocole en mai 2002 et a établi une directive européenne qui fixe les quotas d'émission pour chaque pays membre de l'Union. L'Australie, longtemps alignée sur la position américaine, a ratifié le protocole en décembre 2007. Avec l'Australie, c'est au total 175 pays qui ont adhéré au protocole et sont donc tenus de respecter leurs engagements entre 2008 et 2012. Les Etats-Unis sont le seul grand pays développé qui n'a pas ratifié ce protocole, comme quelques pays d'Afrique (fig. 2). Suite à la ratification australienne, la pression va sans doute s'accentuer sur les Etats-Unis afin qu'ils ratifient ce protocole. Soucieux de redorer l'image internationale des Etats-Unis, le nouveau président Américain, élu pour la période 2009-2012, pourrait engager le processus de ratification de ce protocole, ou coopérer de manière constructive à l'élaboration du protocole post-Kyoto (ou Kyoto II).

En dépit de la très grande adhésion des pays au protocole de Kyoto, il subsiste un pessimisme quant à la volonté politique et à la capacité de la communauté internationale de lutter efficacement contre le réchauffement du climat. Nombreux sont ceux qui estiment qu'il faut travailler non plus seulement sur les moyens de lutte contre ce réchauffement "inéluctable » et «irréversible», mais aussi et surtout sur les moyens d'adaptation de l'Homme et de la biosphère au «nouveau climat imminent». L'après Kyoto fait l'objet de discussions et de négociations internationales depuis quelques années en vue d'un autre accord pour la période 2013-2017. Les discussions portent notamment sur l'augmentation des engagements de réduction des émissions de gaz à effet de serre des pays développés et sur l'implication des pays en développement et surtout des pays émergeants, cette implication étant largement basée sur des incitations financières, ce qui montre l'importance que prend le marché dans les 
décisions censées réduire le changement climatique (Quenault, 2006 ; Karsenty et Pirard, 2007a et b ; Tsayem, 2008).

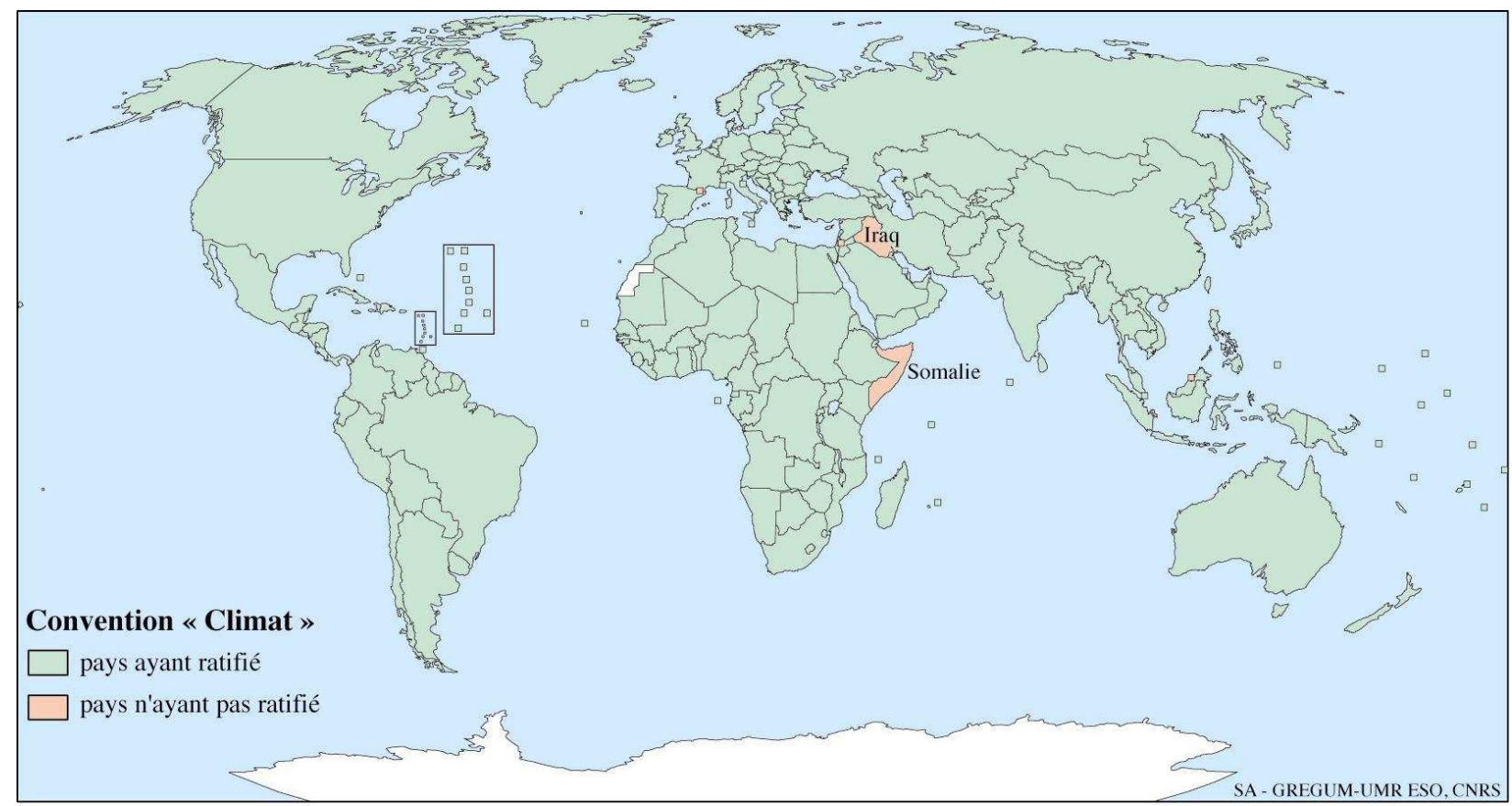

Figure 1. Etat des ratifications de la convention sur le changement climatique (janvier 2008)

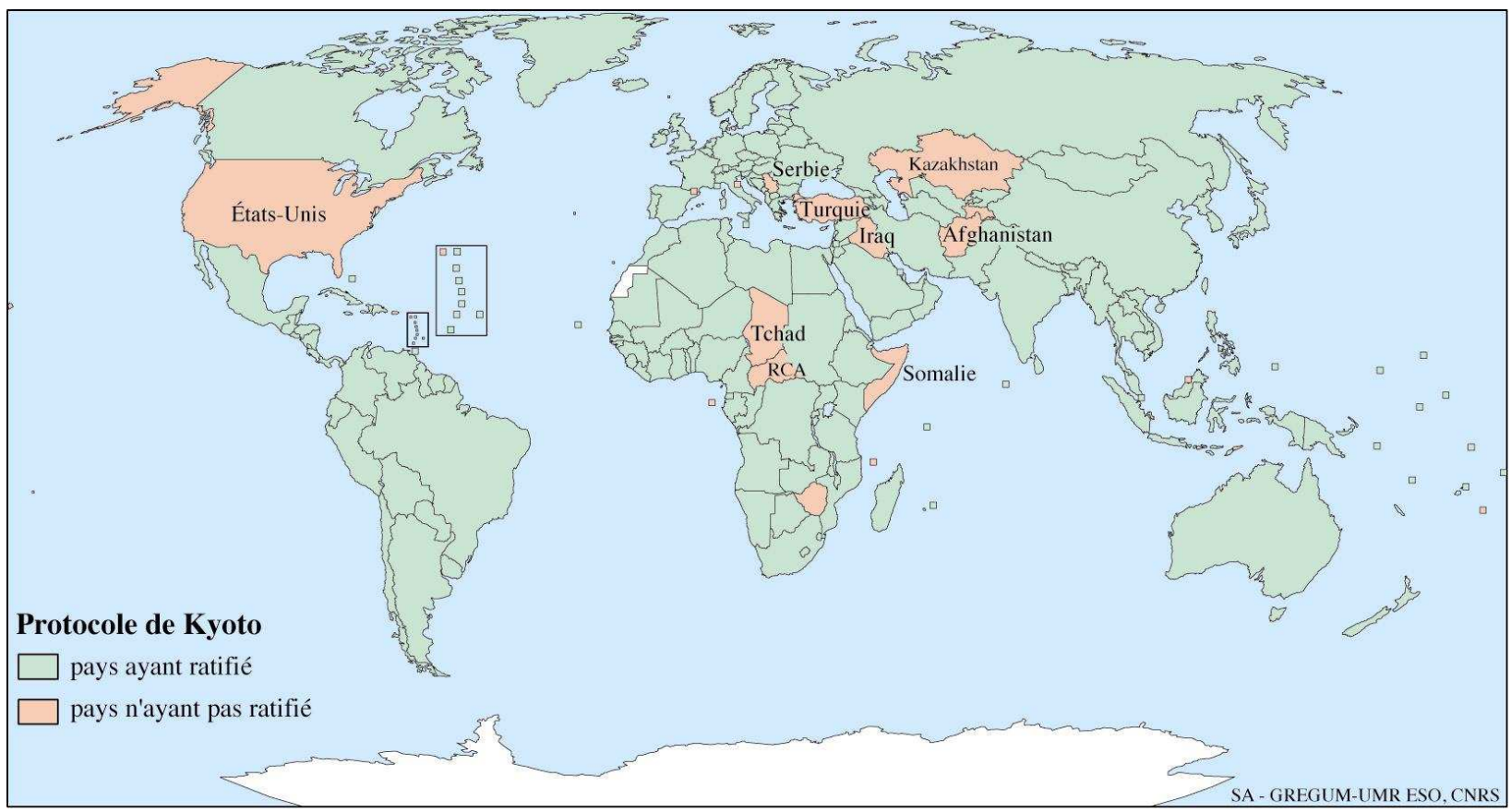

Figure 2. Etat des ratifications du protocole de Kyoto (janvier 2008) 


\section{La Convention sur la diversité biologique : conserver la biodiversité pour exploiter son potentiel économique et industriel}

Cette convention fixe le cadre international pour la protection, l'utilisation et la gestion de la biodiversité suivant les préoccupations relatives au développement durable énoncées notamment en 1987 dans le rapport Brundtland et réaffirmées au sommet de la Terre à Rio. Dans l'article 2 de la convention, la diversité biologique est définie comme étant «la variabilité des organismes vivants de toute origine, y compris, entre autres, les écosystèmes terrestres, marins et autres écosystèmes aquatiques et les complexes écologiques dont ils font partie ». Trois niveaux de biodiversité sont retenus: le niveau des espèces (diversité spécifique), le niveau intra-spécifique (diversité génétique) et le niveau des écosystèmes (diversité écosystémique).

Les objectifs visés par la convention sont énumérés dans l'article 1: parvenir à la conservation de la diversité biologique, à l'utilisation durable des composantes de cette diversité biologique et au partage juste et équitable des ressources génétiques. Il est précisé que ce partage «juste et équitable » doit se faire grâce à un accès "satisfaisant » aux ressources génétiques et au transfert approprié des «techniques pertinentes» en tenant compte des droits sur ces ressources et techniques. Par ailleurs, l'article 1 ajoute que ces objectifs doivent être atteints grâce à un financement "adéquat ». L'article 2 définit deux types de conservation des espèces : la conservation in situ (dans le milieu naturel où les caractères distinctifs des espèces se sont développés) et la conservation ex situ (en dehors du milieu naturel d'origine). Ces objectifs paraissent quelque peu vagues et dénotent du passage de la biodiversité du statut d'objet du patrimoine naturel au statut de bien à exploiter économiquement et industriellement.

La convention stipule que les États ont «le droit souverain d'exploiter leurs propres ressources selon leur politique d'environnement et ont le devoir de faire en sorte que les activités exercées dans les limites de leur juridiction ou sous leur contrôle ne causent pas de dommage à l'environnement dans d'autres États ou dans des régions ne relevant d'aucune juridiction nationale » (art. 3). En reconnaissant à chaque pays le droit souverain de gérer comme bon lui semble les ressources qui se trouvent sur son territoire (d'après sa politique environnementale), la convention apparaît contradictoire par rapport à la considération selon laquelle la biodiversité est « un bien commun de l'humanité, un patrimoine mondial, etc. ».

Les pays signataires de la convention s'engagent soit à élaborer des stratégies, des plans ou des programmes nationaux pour la conservation et l'utilisation durable de la biodiversité, soit à adapter les stratégies, plans ou programmes déjà existants (art. 6).

De nombreux articles de la convention appellent à la prise en compte des besoins spécifiques des pays en développement notamment en matière de financement de la conservation in situ et ex situ (articles 8-m et 9-e), de recherche scientifique et de formation sur la biodiversité (article 12), d'accès à la biotechnologie et à son transfert (art. 16).

L'article 20 de la convention demande explicitement aux pays développés de fournir des ressources financières nouvelles et additionnelles aux pays en développement afin que ces derniers puissent s'acquitter de leurs obligations en matière de conservation et d'utilisation durable de la biodiversité. L'alinéa 4 de cet article précise que le développement économique et social ainsi que l'élimination de la pauvreté sont les «priorités premières et absolues » des pays en développement, ce qui entérine en quelque sorte le principe suivant lequel lorsqu'un pays est pauvre, il ne devrait pas se préoccuper de la protection de l'environnement !

Adoptée à Rio en 1992 et entrée en vigueur en 1993, cette convention a été ratifiée par 189 pays (fig. 3). Les États-Unis l'ont signée mais ne l'ont pas ratifiée, ce qui leur laisse la liberté 
de ne pas respecter les engagements énoncés par la convention. Ils ont justifié leur position en invoquant des questions d'emplois, de protection des droits de propriété intellectuelle et des restrictions liées à l'industrie des biotechnologies.

La lecture de la convention donne l'impression qu'une grande importance a été accordée à l'utilité ou à la valeur économique et industrielle de la biodiversité et des biotechnologies et ce au détriment de la préservation des écosystèmes en tant que habitat des espèces de faune et de flore. Le caractère souple de la convention, résultant sans doute de la volonté de conciliation des points de vue divergents, se traduit par l'utilisation répétitive des expressions « si possible », «si nécessaire », «en cas de besoin », «sous réserves de », « selon qu'il conviendra »...En somme, un traité peu contraignant quant au respect des engagements pris par les États qui le ratifient.

La convention sur la diversité biologique s'ajoute à une panoplie de traités internationaux relatifs à la protection de l'environnement et des espèces de faune et de flore : convention de Ramsar en 1971 sur les zones humides d'importance internationale, convention de Paris en 1972 sur la protection du patrimoine mondial culturel et naturel (UNESCO), convention de Washington en 1973 sur le commerce international de la faune et de la flore menacées d'extinction (CITES), convention de Bonn en 1979 sur la conservation des espèces migratrices appartenant à la faune sauvage, etc. La convention sur la diversité biologique n'a pas réussi le pari d'englober ces conventions précédentes afin de constituer un document juridique unique traitant des questions de biodiversité à l'échelle planétaire. De ce fait, les actions menées par la communauté internationale en vue de la protection, de la conservation et de la gestion dite durable des écosystèmes paraissent éparses et quelque peu incohérentes.

\section{De la diversité biologique aux organismes génétiquement modifiés : le Protocole de Carthagène}

Faisant suite à la convention sur la biodiversité, le protocole de Carthagène sur la biosécurité a été adopté à Montréal en janvier 2000. Il porte sur les organismes vivants modifiés (OVM) ou organismes génétiquement modifiés (OGM) et préconise la prévention des risques biotechnologiques. Ce protocole réglemente les transferts internationaux (exportations), le transit, la manipulation et l'utilisation des OVM (végétaux, animaux et microbes génétiquement modifiés) qui pourraient avoir des effets néfastes sur la conservation et l'utilisation de la diversité biologique et comporter des risques pour la santé humaine. Il exige que l'exportation et/ou l'importation des organismes vivants modifiés (maïs ou soja par exemple), destinés à l'alimentation humaine et animale, ou destinés à être transformés, soient accompagnée de documents d'expédition indiquant que ces produits «peuvent contenir » des organismes génétiquement modifiés et qu'ils ne sont pas destinés à être introduits intentionnellement dans l'environnement.

Entré en vigueur en septembre 2003, ce protocole illustre bien le fait que sur le plan international, la valeur économique et industrielle de la biodiversité a pris le pas sur les objectifs de conservation de cette biodiversité. On peut alors s'interroger sur le bien-fondé et les enjeux des discours internationaux sur la protection de la nature : pourquoi protéger les milieux et les espèces ? On pourrait répondre de manière caricaturale en disant que c'est pour mieux les exploiter (en raison de leur rentabilité commerciale et industrielle). Ce protocole mobilise peu la communauté internationale. Seuls 143 pays l'ont ratifié (fig. 4). 


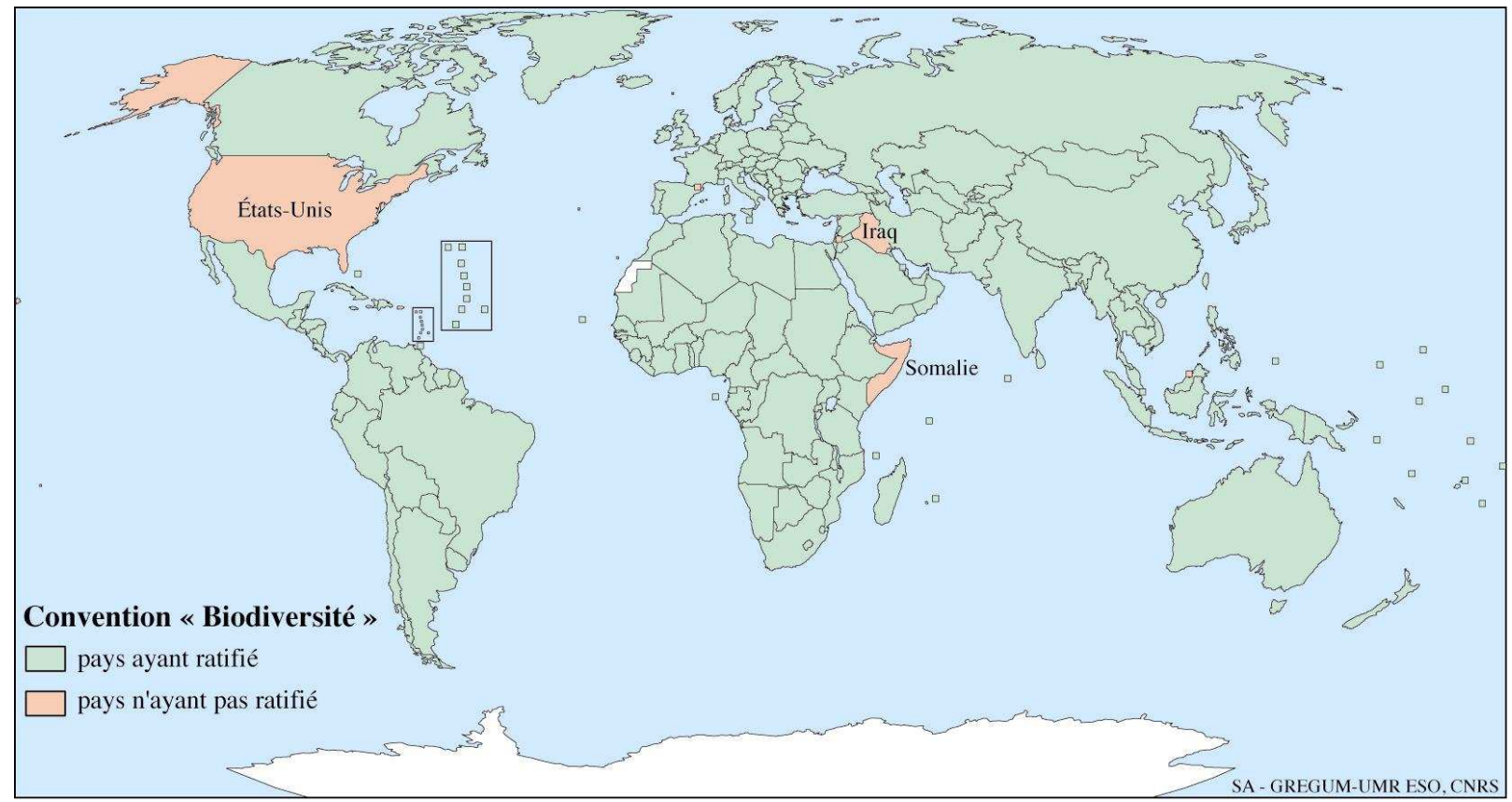

Figure 3. Etat des ratifications de la convention sur la diversité biologique (janvier 2008)

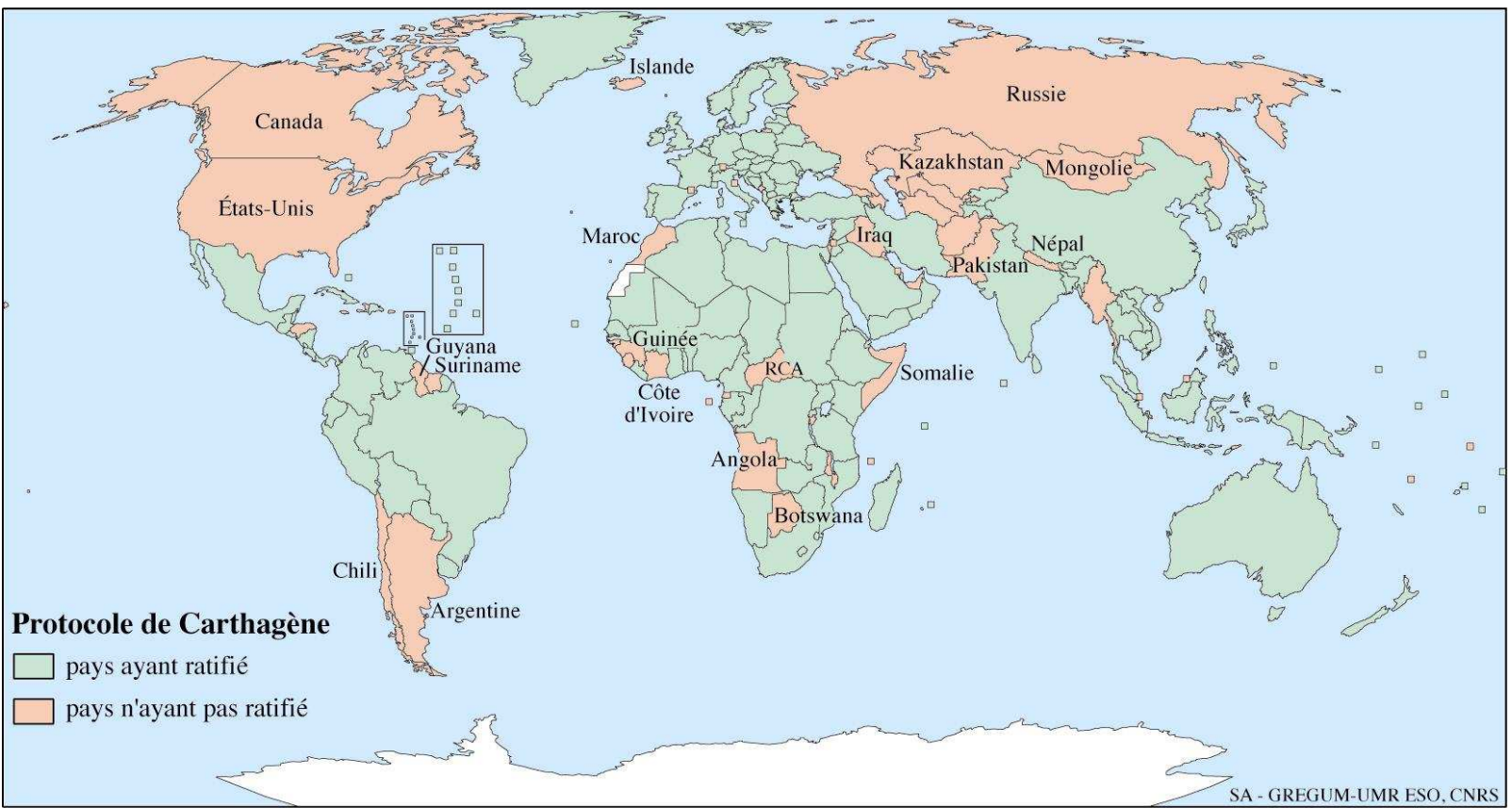

Figure 4. Etat des ratifications du protocole de Carthagène (janvier 2008)

\section{La Convention sur la lutte contre la désertification et la sécheresse : le parent pauvre}

A la demande des pays touchés par ces phénomènes, la lutte contre la sécheresse et la désertification avait été inscrite à l'ordre du jour de la conférence de Rio en 1992. Les discussions sur le sujet n'ayant pas abouti à un traité à Rio, il a été demandé aux Nations 
unies de mettre en place un comité intergouvernemental de négociations. Ses travaux ont abouti à la rédaction du texte de la convention adoptée à Paris en 1994 et ouverte aux signatures et aux ratifications.

D'après l'article 1 de la convention, la désertification est la «dégradation des terres dans les zones arides, semi-arides et subhumides sèches par suite de divers facteurs, parmi lesquels les variations climatiques et les activités humaines ». Quant à la sécheresse, c'est « le phénomène naturel qui se produit lorsque les précipitations ont été sensiblement inférieures aux niveaux normalement enregistrés et qui entraîne de graves déséquilibres hydrologiques préjudiciables aux systèmes de production des ressources en terre » (article 1-c).

L'objectif principal de la convention est «de lutter contre la désertification et d'atténuer les effets de la sécheresse dans les pays gravement touchés par la sécheresse et/ou la désertification, en particulier en Afrique, grâce à des mesures efficaces à tous les niveaux, appuyés par des arrangements internationaux de coopération et de partenariat, dans le cadre d'une approche intégrée compatible avec le programme Action 21, en vue de contribuer à l'instauration d'un développement durable dans les zones touchées » (article 2, alinéa 1). L'alinéa 2 de l'article 2 précise qu'il faudra appliquer des stratégies « intégrées à long terme » axées sur l'amélioration de la productivité des terres, la restauration, la conservation et la gestion durable des ressources en terre et en eau, ceci afin d'aboutir à l'amélioration des conditions de vie des collectivités vivant dans les régions touchées.

Curieusement, la convention demande aux pays touchés d'accorder la priorité «voulue » à la lutte contre la désertification et à l'atténuation de la sécheresse et d'y consacrer des ressources suffisantes en rapport avec la situation et les moyens disponibles (article 5-a). Quant aux pays développés, il leur est demandé, comme dans les autres conventions et protocoles présentés dans ce document, de fournir des ressources financières importantes et d'autres formes d'appui pour aider les pays en développement touchés (article 6-b).

La convention demande que la priorité soit accordée à l'Afrique (article 7), continent le plus touché par la désertification et la sécheresse et ne bénéficiant pas d'une situation économique lui permettant de lutter contre ces fléaux.

Entrée en vigueur en 1996, cette convention a été ratifiée par 190 pays (fig. 5). Cependant, on a l'impression qu'elle est en léthargie et mobilise peu la communauté internationale. Les engagements préconisés par la convention paraissent très souples et peu contraignants. N'y a t-il pas un lien entre la faible mobilisation de la communauté internationale et le fait que la sécheresse et la désertification touchent surtout les pays en développement, pauvres en général et politiquement faibles ? Pourtant, avec le changement climatique, les experts, et notamment ceux du GIEC ( $4{ }^{\text {ème }}$ rapport), annoncent une exacerbation de la sécheresse et de la désertification.

A ce jour, la convention sur la lutte contre la désertification souffre toujours de l'absence ou de la très faible disponibilité des ressources financières pour sa mise en œuvre dans les pays touchés. Ces pays avaient souhaité en vain que la convention bénéficie d'un mécanisme international de financement comme c'est le cas avec le Fonds de l'Environnement Mondial qui contribue financièrement au fonctionnement des conventions sur les changements climatiques et sur la diversité biologique. Contrairement aux conventions sur le changement climatique et sur la diversité biologique, la convention sur la sécheresse et la désertification n'a pas été complétée par un protocole. 


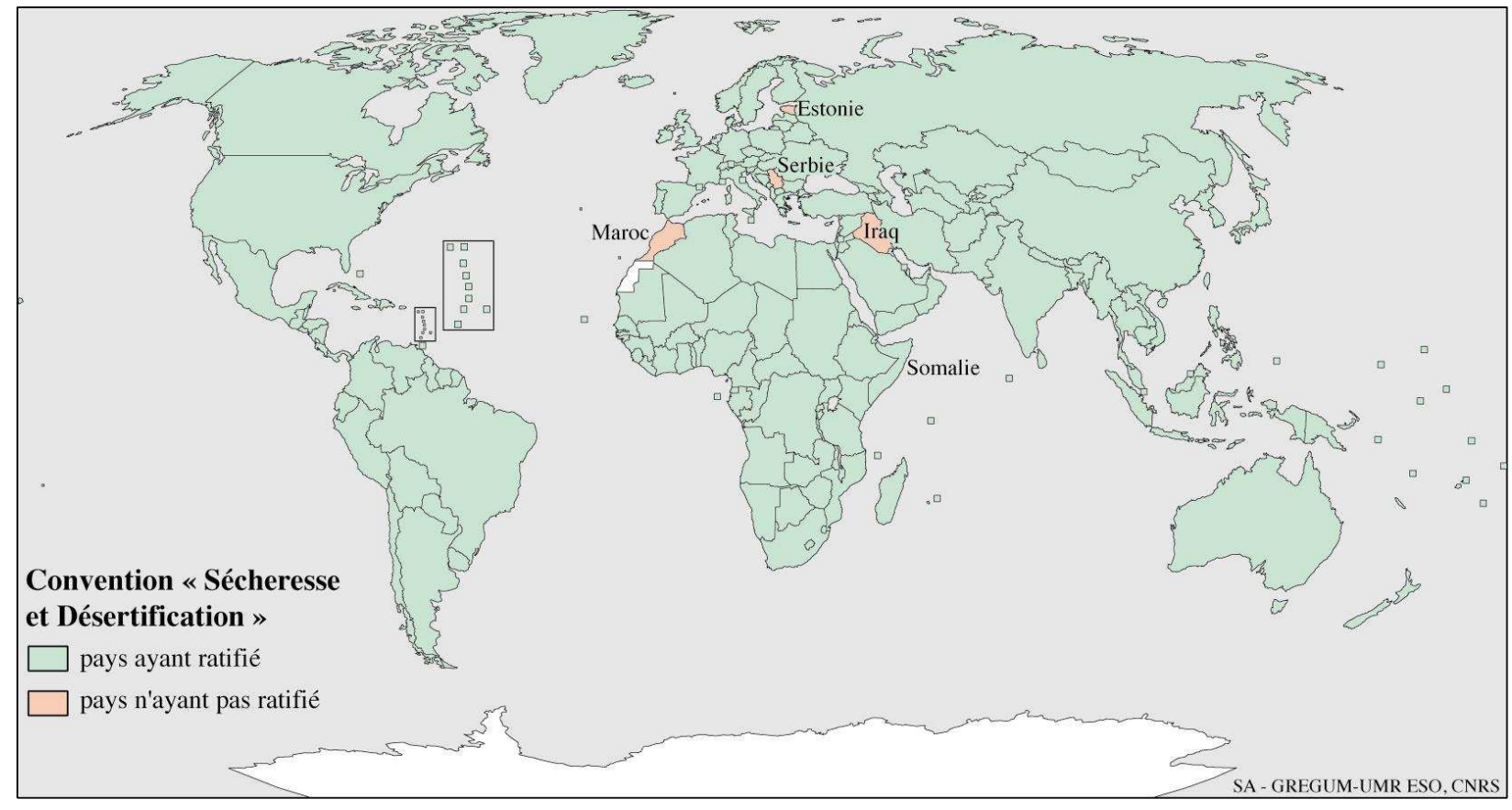

Figure 5. Etat des ratifications de la convention sur la sécheresse et la désertification (janvier 2008)

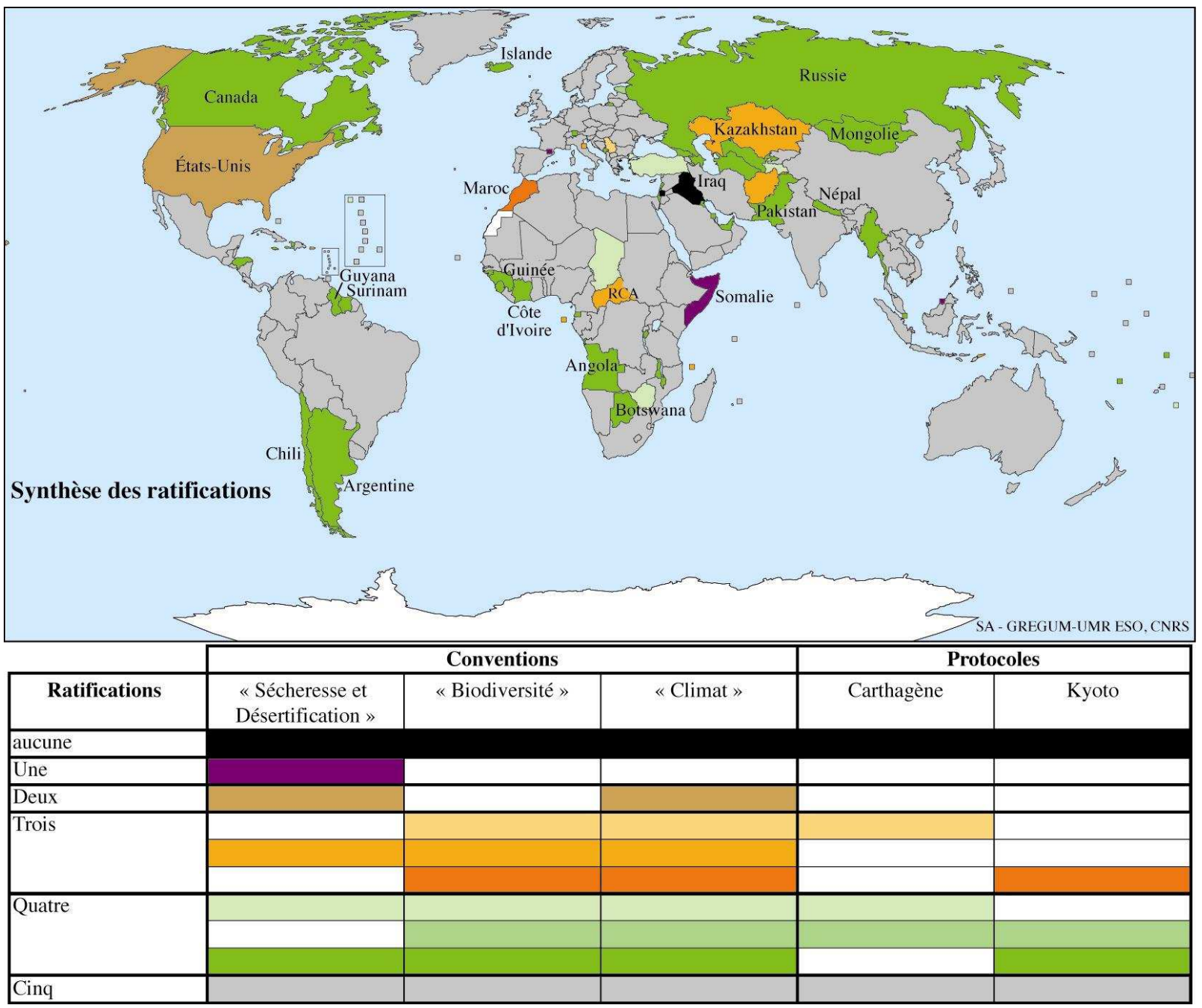

Figure 6. Synthèse des ratifications (janvier 2008) 


\section{Essai de synthèse des ratifications : les intérêts nationaux confrontés aux enjeux internationaux ou mondiaux}

Afin de comparer les engagements des Etats dans le cadre des conventions et protocoles présentés dans ce document, une synthèse des ratifications a été réalisée sous forme de représentation cartographique. Elle permet de visualiser successivement les pays qui n'ont ratifié aucune des trois conventions ni aucun des deux protocoles, puis les pays qui ont ratifié seulement une convention ou un protocole, les pays qui en ont ratifié deux, trois, quatre et enfin l'ensemble des cinq textes (fig. 6). Cette carte montre que les trois conventions et les deux protocoles, considérés collectivement, sont aujourd'hui en vigueur dans 134 Etats. En d'autres termes, parmi les pays membres du système des Nations unies, 134 ont ratifié aussi bien les conventions sur le climat, sur la biodiversité, sur la sécheresse et la désertification, que les protocoles de Kyoto et de Carthagène. 37 pays ont ratifié les 5 textes moins le protocole de Carthagène. 4 pays n'ont ratifié aucun de ces textes (cas de l'Iraq), tandis que 3 pays n'ont ratifié qu'un seul texte (cas de la Somalie qui n'a ratifié que la convention sur la sécheresse et la désertification). Un seul pays n'a ratifié que 2 des 5 textes. Il s'agit des EtatsUnis qui ont ratifié seulement la convention sur la sécheresse et la désertification et la convention sur le climat.

Beaucoup d'Etats ont été prompts à ratifier ces conventions et protocoles (Etats qu'on peut qualifier de «bons élèves »). Il s'agit essentiellement de pays en développement et de pays émergents qui ont un traitement de faveur dans ces traités internationaux sur l'environnement. Il apparaît dès lors que la ratification par ces Etats ne signifie pas nécessairement qu'ils sont plus respectueux ou plus soucieux de l'environnement que les «mauvais élèves » (Etats comme les Etats-Unis ou le Canada, qui sont réticents à ratifier ces traités, sans doute pour ne pas être tenus par des obligations internationales contraignantes). Cette situation est révélatrice du jeu politique des Etats en matière de relations internationales, y compris dans le domaine de l'environnement. Là aussi, les intérêts nationaux sont pesés par rapport à l'intérêt mondial ou global, et les Etats s'engagent d'autant moins promptement que des contraintes internationales apparaissent pesantes par rapport aux intérêts nationaux.

\section{Conclusion}

S'inscrivant dans la mouvance du paradigme du développement durable qui se répand dans le monde en particulier depuis le sommet de Rio de Janeiro en 1992, les conventions internationales sur l'environnement présentées dans ce document témoignent des tentatives d'engagement et d'organisation de la communauté internationale en vue d'apporter des réponses aux problèmes environnementaux contemporains. Ces traités associent les pays développés et les pays en développement en faisant en sorte que les pays développés « assument leur responsabilité historique » dans la dégradation de l'environnement planétaire. Ainsi, les efforts les plus importants, tant sur le plan du financement de la lutte contre la dégradation de l'environnement que sur le plan des mesures à mettre en ouvre en vue d'améliorer l'état de l'environnement mondial, sont demandés essentiellement aux pays développés. Bien que ces conventions aient suscité l'adhésion de la quasi-totalité des pays du monde (à l'exception notable des Etats-Unis pour ce qui concerne le protocole de Kyoto et la convention sur la diversité biologique), les engagements des pays paraissent minimalistes et insuffisants par rapport à l'ampleur et à la gravité de ces problèmes telles que cette ampleur et cette gravité sont rappelées ou proclamées dans ces traités. Les négociations internationales qui se tiennent régulièrement dans le cadre du fonctionnement et du suivi de ces conventions essaient d'augmenter, pour les années à venir, les niveaux d'engagement et d'implication des pays développés mais aussi des pays en développement et surtout des pays émergeants. L'implication des pays développés et des pays émergeants, avec des engagements chiffrés 
contraignants, est envisagée en particulier pour le protocole de Kyoto à l'horizon 2013 (deuxième phase d'application dite Kyoto II). En adoptant le principe de la «déforestation évitée », qui complète le «mécanisme pour un développement propre », la Conférence de Bali en décembre 2007, organisée par l'ONU dans le cadre de la convention sur le changement climatique, a entériné le fait que les pays du Sud, pays en développement et pays émergeants compris, devront prendre des engagements en vue de participer aux efforts nécessaires pour résoudre les problèmes environnementaux mondiaux (Karsenty et Pirard, 2007a et b). Le fait que cette participation des pays du Sud soit inscrite essentiellement dans le cadre des mécanismes de flexibilité sous-tendus par des considérables financières (marchés du carbone et des quotas d'émissions, compensations financières, payements pour services environnementaux) montre que la lutte contre le réchauffement de la Terre (et plus globalement la protection de l'environnement) est de plus en plus soumise au marché et à la logique économique marchande (Tsayem, 2008). On peut s'interroger sur l'efficacité à long terme de cette vaste entreprise de «sauvegarde » de l'environnement planétaire si les mesures à prendre le seront à l'aune du prix de la tonne de gaz carbonique dans les marchés mondiaux. Au-delà de la mobilisation des Etats au travers des ratifications, se pose la question de l'efficacité de ces conventions dont les fondements théoriques sont difficiles à saisir et dont l'application est largement inspirée et inscrite dans les mécanismes marchands en effervescence depuis la création des marchés carbone.

\section{Références bibliographiques}

Durand F., 2002. Au royaume des aveugles, l' «effet de serre» est roi. In Le Monde Diplomatique, décembre 2002.

Quenault B., 2006. Protocole de Kyoto et gouvernance écologique mondiale : enjeux et perspectives des engagements post-2012. Mondes en Développement, $\mathrm{n}^{\circ} 136$, p.29-47.

GIEC, 2001 (3ème rapport). Changement climatique 2001. Rapport de synthèse. Résumé à l'intention des décideurs. $37 \mathrm{p}$.

Karsenty A., Pirard R., 2007a. Changement climatique: faut-il récompenser la «déforestation évitée » ? Natures Sciences Sociétés, ${ }^{\circ}$ 15, p. 357-369.

Karsenty A., Pirard R., 2007b. Forêts tropicales : la question du bien public mondial et la quête d'instruments économiques multilatéraux pour un régime international. Revue Forestière Française, $\mathrm{n}^{\circ}$ 5, p. 535-547.

GIEC, 2007 (4eme rapport). A report of working group I of the intergovernmental panel on climate change. Summary for policymakers, $18 \mathrm{p}$.

Tsayem Demaze M., 2009a. Paradoxes conceptuels du développement durable et nouvelles initiatives de coopération Nord-Sud : le mécanisme pour un développement propre (MDP). CyberGeo, Revue Européenne en Géographie, article 443 (http://www.cybergeo.eu/index22065.html).

Tsayem Demaze M., 2009b. Le protocole de Kyoto, le clivage Nord-Sud et le défi du développement durable durable. L'Espace Géographique, $\mathrm{n}^{\circ}$ 2, p. 139-156.

Tsayem Demaze M., 2008. Le business du développement durable dans les pays du Sud: quand la lutte contre le changement climatique crée un marché alimenté par le $\mathrm{CO}_{2}$. Communication présentée au séminaire de l'UMR ESO, axe Espaces et Territoires de la Gouvernance, Le Mans, le 17 novembre 2008. 